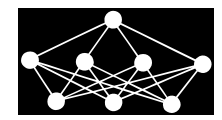

\title{
MEMORY BASED DECISION MAKING: A SPIKING NEURAL CIRCUIT MODEL
}

\author{
Min Xia*, Chong Zhang ${ }^{\dagger}$ Yin Wang ${ }^{\dagger}$ Jia Liu, Chunzheng Li ${ }^{\dagger}$
}

\begin{abstract}
Conscious decision making is one of the important functions of human behavior. Episodic memory is the source of knowledge for conscious decision making. The mechanism of how episodic memory affects conscious decision-making is unclear. To investigate the brain mechanism of conscious decision making, we investigated a biologically-based network model of spiking neurons for competition between automatic response and conscious decision making. The proposed model integrates episodic memory modular and brain decision-making modular, and uses episodic memory output as the top-down input of decision making. In the decision making, the network realizes the competition between decision patterns through mutual inhibition, finally reaches the conscious decision making. The simulations show that the proposed model can well implement multimodal coherent decision making under sequential memory control. The proposed model can effectively explain the transmission mechanism of conscious decision information.
\end{abstract}

Key words: decision making, sequence memory, top-down control, spiking neural circuit

Received: March 25, 2018

DOI: $10.14311 /$ NNW.2019.29.010

Revised and accepted: June 26, 2019

\section{Introduction}

Brain decision making is one of the most important neural activities in human beings. Automatic response and conscious decision making are two important representations of brain decision-making [1,2]. Automatic response is a rapid decisionmaking process, such as touching a hot object and retracting your hand quickly. Conscious decision making is relatively slow, conscious decision-making mainly uses the existing knowledge to guide decision-making, and episodic memory is the source of knowledge for conscious decision-making [3-6]. The flexibility of behavior activity is an important sign of human decision-making ability, which requires

\footnotetext{
*Min Xia - Corresponding author; Jiangsu Key Laboratory of Big Data Analysis Technology; Jiangsu Collaborative Innovation Center on Atmospheric Environment and Equipment Technology, Nanjing University of Information Science and Technology, Ningliu Road 219, Nanjing, China, 210044, E-mail: xiamin@nuist.edu.cn

${ }^{\dagger}$ Chong Zhang, Yin Wang, Jia Liu, Chunzheng Li, Jiangsu Key Laboratory of Big Data Analysis Technology, Nanjing University of Information Science and Technology, Ningliu Road 219, Nanjing, China, 210044.
} 
the brain to solve the conflict between automatic response and conscious decision making $[7,8]$.

The brain is a complex autonomous system. It is necessary to establish a complex network model to analyze the correlation between neural structures [9]. In the past few years, a large number of experimental data related to brain decisionmaking have been accumulated through experiments. In recent years, some researchers have proposed models related to brain decision-making system by analyzing these experimental data. Wang [10] proposed a mutual inhibition network model of visual discrimination decision, the model established two excitatory neuron clusters, each neuron clusters represent a decision, the two excitatory neurons compete with each other mediated by an inhibitory neurons cluster. Under the combined action of external stimulus, one decision from two choices is done. This model is considered to be one of the pioneering work in the research of brain decision-making. Based on this work, Machens [11] proposed a working memory information flexible inhibition control model. By comparing the stimulus and memory, the model controls the competition between decision neurons, and realizes the simple memory decision association. In 2006, Deco [12] realized the expression of decision making under Webb's law by using the mutual inhibition network model. Koster [13] realized the modeling of eye movement cortex control decision based on mutual inhibition network structure, the directional (Top-down) control signals are generated through the directional selectivity of visual neurons to guide eyelid movement. Cain [14] used the neural dynamics model to analyze the parameters required for the brain decision network in detail. Based on these models, some scholars have also studied the influence of the Top-down control signal strength on the decision speed and accuracy $[15,16]$.

In recent years, researchers have paid more and more attention to the relationship between memory and decision making [17-23]. Hikosaka [18] used basal ganglia striatal suppression mediated modeling to achieve a switch between automatic response and conscious decision making. Fechner [19] constructed a computational model of neural dynamics through the Adaptive Control of Thought-Rational (ACT-R) cognitive architecture, and realized the top-down control of memory for decision making. Khader's model [20] illustrated the role of automatic and controlled activation of neural clusters in the balance between automatic response and conscious decision making. This automatic and controlled activation is associated with the activation of memory patterns in the prefrontal cortex network. Bogacz and Wei's works [21,22] indicated that basal ganglia play an important role in information transduction in conscious decision-making, and basal ganglia can be used for decision making through the inhibitory effect of neural circuits. The study of Chung [7] indicated that the decision making competition mechanism of the superior colliculus is a key step in the implementation of conscious decision-making.

Up to now, people have made a macroscopic understanding of the structure and function mechanism of brain decision-making system through the research of brain decision biology experiments. Through the study of neural dynamics model, we can understand the mechanism of neural cluster in different brain regions in brain decision-making system from the point of view of mechanism modeling. These studies have laid an advanced model structure for further understanding about human brain decision-making processes. Machens [11] suggested that the final re- 
alization of the artificial brain must rely on accurate neural system modeling. A large number of biological experiments show that episodic memory model can be mapped into decision model space by synaptic plasticity learning. The competition mechanism between knowledge based decision making and stimulus response is the way to realize conscious decision making. However, the current research focuses on the expression of decision information, but rarely involves the transmission mechanism of conscious decision-making information. The rapid development of biological experiments on the brain neural decision need the neural dynamics mechanism model to explain the related phenomenon.

A model for mapping sequence memory into decision space is proposed in this paper. The model integrates sequence memory model and brain decision-making model, and uses sequence memory output as the top-down input of decision making. At the same time, the external world stimulus decision neurons. In the process of decision-making, there is competition between decision patterns, and the network finally reaches the winner-take-all. Only one or several of these patterns wins the competition by increasing its activity to a threshold, while the other patterns are suppressed. This model effectively explains the transmission mechanism of conscious decision information.

In this work, the main contribution is to associate brain sequential memory with brain decision-making, to establish conscious decision-making model, that is, to propose a new neural network decision-making structure based on current biological conclusions. Based on the model, the output of the sequential memory model is used as top-down input of the decision model, and sensory stimuli is input from the outside, above two signals make the decision patterns compete with each other. Decision-making patterns associated with memory patterns win the competition by increasing their own firing rates, while other decision-making patterns are suppressed. Different from the existing decision-making models, which mainly focus on eye-jump stimulation decision-making of simple tasks, the brain memory model in this paper can guide decision-making and achieve multi-task decision-making. In this model, the effects of both external stimulus (unconscious) and memory pattern (active consciousness) on decision-making are considered simultaneously.

\section{Materials and methods}

\section{$2.1 \quad$ Neural architecture}

The hypothetical premises of the neural network structure and the neuron model in this paper are based on the existing biological experimental conclusions. In the proposed network, the full neural circuit includes two brain areas: the prefrontal cortex for memory, and the superior colliculus for decision making. The memory is the up-down control signal for decision making. The conceptual overview of the network model is shown in Fig. 1. In this work, the integrate-and-fire framework introduced by Brunel [24] is used to realize the neural spiking dynamics. The parameters of neurons in this paper are obtained from biological experiments [25]. The computational model of the memory was described by Deco previously [26,27]. The network model used different specific pools for memory patterns. As shown in Fig. 1 , the specific pools are labeled by $P_{1}, P_{2}, \ldots, P_{n}$, each pool represents a 


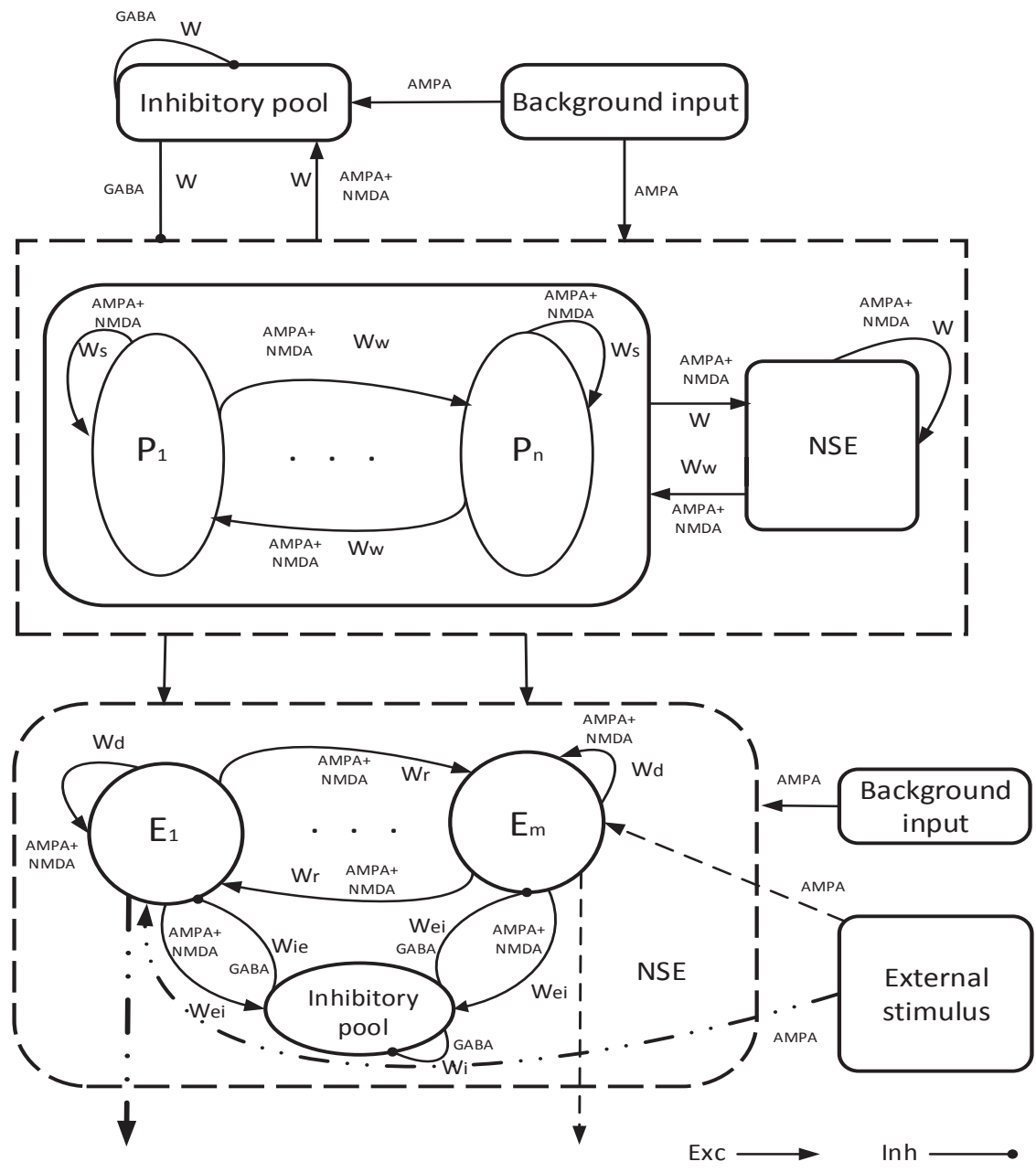

Fig. 1 The structure of model.

pattern, and the specific pools are activated sequentially. In the memory model, different specific pools represent different items of sequence memory (e.g., a simple motor action, visual identification, etc.). The experimental results [28] indicate that competitive firing in hippocampus is the physiological basis of sequence memory. In the neural system that generates sequence memory, excitatory synapses and inhibitory synapses coexist. For example, in hippocampus and many areas of cerebral cortex, sequence memory is produced by the interaction of excitatory and inhibitory neuronal clusters through synaptic connections. In the sequence memory model, the excitatory pool is divided into two parts: specific pool and nonspecific pool. The specific pool is used for memory coding, and the nonspecific pool is used to introduce some noise into the network [29]. The shunting inhibition is used in 
the model for maintaining stability of the network, leading to the low firing rate of the network [24]. In the memory model, the adaptation mechanism is applied in neural pool when it is activated, and then it will create the global inhibition signal to shut down the previous activate specific pool, leading to the emergence of the next pattern. Thus, when a short sequence of stimuli injected into the network, the network will remember them in order.

In the sequence memory part, the network includes $N_{E}$ excitatory pyramidal neurons and $N_{I}$ inhibitory interneurons. Biological experiments indicate that the pattern of sequence memory is stored in excitatory neuron clusters, and different patterns are stored in different excitatory neuron clusters. The ratio of excitatory and inhibitory neurons is $8: 2$, according to the physiologically observation by Abeles [30]. There are $f N_{E}$ neurons for each specific pool, where $f$ is the fraction of the specific neurons, and it is set as $f=10 \%$. Thus, the remaining $N_{E}-n \cdot f N_{E}$ specific neurons are in the nonspecific pool. In order to make the proposed model closer to the mechanism of real situational memory, the noise induced by non-coding neurons firing appears Poisson distribution. The inhibitory pool in the network is applied to create the global competition throughout the memory network, that the sequence can be achieved. In the simulation, the number of the pyramidal neurons and inhibitory interneurons is $N_{E}=800$ and $N_{I}=200$ respectively. In the sequence memory processing, the synaptic connection strength between two neurons follows the Hebbian learning rule, which can realize the transformation from short-term memory to long-term memory [31]. Thus, the correlated activity will increase the coupling strength between the neurons. Following this rule, the coupling weight between the neurons in the specific pool is set as $W_{s}=3.2$. The coupling strength between two neurons from different specific pools is weaker than that in same specific pools, and it is given by $W_{w}=1-f\left(W_{s}-1\right)(1-f)$. The feed-forward synaptic weight from specific excitatory pool to nonselective pool is given by $W=1$, while the weight for feedback synaptic connection is $W_{w}$. The coupling weight between the interneurons is $W=1$, and the interneurons connect all specific neurons with same weight $W=1$. In the neural system, a large number of neurons form neural circuits through synaptic connections. The coding neurons are connected by NMDA receptors and AMPA receptors. Inhibitory neurons globally inhibit coding and non-coding neurons through GABA receptors. Noncoding neurons and coding neurons are connected by excitatory receptors NMDA and AMPA.

The network structure of brain decision-making is also derived from existing biological experiments. Shadlen's results [32] show that brain decision-making is based on existing memory patterns, and memory is the Top-down signal for decision-making. Khader's experiments [20] show that the activation and control sequence of neuronal regions associated with decision-making options can be tracked in material-specific brain regions. This indicates that the brain decisionmaking model is stored in different neuron regions, which is the empirical basis of the brain decision-making network model in this paper. The neural circuit for decision making draws on previous works [33]. The network for decision making consists three neural populations: excitatory coding pools $E_{i}, i=1,2, \ldots, m$ selective for choice alternatives, nonselective excitatory neurons $N S E$, and the inhibitory interneurons $I_{d}$. In the simulation, the number of neurons in each coding 
pool is 240 , which represents the decision selectivity. The inhibitory population is applied to create the global competition among the excitatory coding pools, and it contains 400 inhibitory neurons. There are 1100 excitatory neurons used in NSE pool. Each excitatory coding pool $E_{i}$ receives the stimulus from the memory output of $P_{j}, j=k_{1}, k_{2}, \ldots, k_{s}$, that is to say, multiple memory patterns correspond to a decision (for example, different scenes can make people laugh). The stimulus of $P_{j}$ can be considered as the top-down control signal for $E_{i}$. The decision result exhibits winner-take-all competition through the inhibitory population. When the activity of a excitatory coding pool reach the decision threshold, other pools are suppressed. This phenomenon has been confirmed by biological experiments of monkeys' random-dot task [34]. Sequential memory patterns are connected by excitatory receptors AMPA. Decision-making patterns are connected by AMPA and NMDA receptors. Inhibitory neuron clusters inhibit decision-making patterns by GABA receptors. Decision-making patterns stimulate inhibitory neurons by AMPA and NMDA receptors. If one or more decision-making patterns achieve cluster firing, these neuron clusters will increase the firing rate of inhibitory neuron clusters through AMPA and NMDA receptors, thereby inhibiting other decision-making patterns. In this process, although the activation patterns are also inhibited by inhibitory neuron clusters, the activation patterns will not be inactivated due to the large mutual excitation stimuli within the activation patterns. At the same time, each excitatory coding pool receives the external stimuli through AMPA receptors, which can lead to the unconscious reactions. The synaptic weight between two neurons in same excitatory coding pool is $W_{d}=0.887$. The weight from excitatory neuron to inhibitory interneuron is $W_{e i}=0.5$. The weight from all excitatory neurons to the $N S E$ neurons is $W_{n}=0.493$. The weight from excitatory neurons to a coding pool is $W_{r}=0.44$. The synaptic weight between two neurons in inhibitory pool is $W_{i}=1.1$. The connection weight from inhibitory neuron to excitatory neuron is $W_{i e}=0.97$. Machens's study [35] shows that decision-making is controlled by memory signals, and that action control is achieved by mutual restraint between decision-making patterns. Gallivan's [36] research indicates that brain sequential decision-making is achieved through competition among decision-making patterns, which is accomplished by local inhibition between patterns. The experiments of Kuo's work [1] shows that there is a game relationship between controlled decision-making (memory-based decision-making) and stimulus response. The proposed network structure effectively constructs the game, that is, external stimulus stimulates decision-making mode through excitatory synapses, which makes the Top-down signal stimulus of sequential memory compete with external stimulus. If the external stimulus and the memory signal are consistent, the decision-making will be accelerated. If the external stimulus and the memory signal are inconsistent, then the game between the external stimulus and the memory signal will lead to a slower decision-making speed.

In this work, the synaptic current are mediated by three receptors: AMPA, NMDA, and GABA. Each neuron from prefrontal cortex and superior colliculus receives external inputs mediated by AMPA synaptic connection with Poisson spike rate of $2400 \mathrm{~Hz}$, which serves as the background noise from the outside. The external inputs of Poisson spikes are generated randomly and independently. The recurrent excitatory postsynaptic potentials are mediated by AMPA and NMDA 
receptors. The synaptic currents from inhibitory neurons to all other neurons are mediated by GABA receptors. From the physiological point of view, the memory signal should be mediated by the basal ganglia. In many works, the basal ganglia is modeled as simple direct or hyperdirect pathways, which can adjust the threshold level of the decision $[22,37]$. In this work, we just focus on the how memory influences the choice making, that the basal ganglia is neglected in this work, and the memory output is directly connected to the superior colliculus.

\subsection{Neural circuit model}

In this work, the synaptic connections between neural populations and the recurrent connections are all to all. In this section, the single neuron and synapse are modeled by leaky integrate-and-fire model, which is with conductance-based synapses, following in the previous studies [24,37]. The membrane dynamics of each neuron evolves as following equation:

$$
C_{m} \frac{\mathrm{d} V(t)}{\mathrm{d} t}=-g_{m}\left(V(t)-V_{L}\right)-I_{\mathrm{syn}}(t),
$$

where $I_{\mathrm{syn}}(t)$ is the total synaptic current to the neuron, $C_{m}$ is the total membrane capacitance, $g_{m}$ is total leak conductance, $V_{L}$ is the resting potential. The total leak conductance $g_{m}$ is $25 \mathrm{~ns}$ for pyramidal neurons and $20 \mathrm{nS}$ for interneurons. In this work, the resting potential $V_{L}$ is set as $V_{L}=-70 \mathrm{mV}$ for both excitatory and inhibitory neurons. When the neuron's membrane potential $V(t)$ reaches a threshold $V_{\text {threshold }}=50 \mathrm{mV}$, a spike is generated and the membrane potential $V(t)$ is set to the reset potential $V_{\text {reset }}=-55 \mathrm{mV}$. Then the neuron is unable to spike again for a period $T_{r}$, which is the absolute refractory period. The absolute refractory period is $2 \mathrm{~ms}$ for pyramidal neurons and $1 \mathrm{mS}$ for interneurons.

The total synaptic current $I_{\text {syn }}(t)$ is combined by the external inputs from the outside, background noise, and recurrent inputs from other neurons. All these currents are mediated by three types of synaptic receptors: the excitatory synaptic currents are mediated by AMPA receptors and NMDA receptors, and the inhibitory components are mediated by GABA receptors. Thus, the total synaptic current $I_{\mathrm{syn}}(t)$ to each neuron can be given by:

$$
I_{\mathrm{syn}}(t)=I_{\mathrm{AMPA}, \text { ext }}(t)+I_{\mathrm{AMPA}, \mathrm{rec}}(t)+I_{\mathrm{NMDA}, \text { rec }}(t)+I_{\mathrm{GABA}}(t),
$$

where $I_{\mathrm{AMPA} \text {,ext }}(t)$ is the external stimulus to the neurons. The synaptic currents for different synaptic receptors are described as following:

$$
\begin{aligned}
I_{\mathrm{AMPA}}(t) & =g_{\mathrm{AMPA}} s_{\mathrm{AMPA}}(t)\left(V(t)-V_{E}\right), \\
I_{\mathrm{NMDA}}(t) & =\frac{g_{\mathrm{NMDA}} s_{\mathrm{NMDA}}(t)\left(V(t)-V_{E}\right)}{1+\left[M g^{2+}\right] E^{-0.062 V(t) / 3.57},} \\
I_{\mathrm{GABA}}(t) & =g_{\mathrm{GABA}} s_{\mathrm{GABA}}(t)\left(V(t)-V_{I}\right),
\end{aligned}
$$

where $V_{E}=0$ and $V_{I}=-70$ the reversal potentials for the excitatory neurons and the inhibitory neurons respectively. $g_{\mathrm{AMPA}}, g_{\mathrm{NMDA}}$ and $g_{\mathrm{GABA}}$ are the synaptic conductance strength between the neurons. In this work, the NMDA-mediated 
currents have a voltage dependence controlled by the extracellular magnesium concentration $\left[\mathrm{Mg}^{2+}\right]=1 \mathrm{mM}[38] . s$ is the gating variable denoting the fractions of open channels, which are given by:

$$
\begin{gathered}
\frac{\mathrm{d} s_{\mathrm{AMPA}}(t)}{\mathrm{d} t}=-\frac{s_{\mathrm{AMPA}}(t)}{\tau_{\mathrm{AMPA}}}+\sum_{k} \delta\left(t-t^{k}\right), \\
\frac{\mathrm{d} s_{\mathrm{NMDA}}(t)}{\mathrm{d} t}=-\frac{s_{\mathrm{NMDA}}(t)}{\tau_{\mathrm{NMDA}, \text { decay }}}+\alpha x(t)\left(1-s_{\mathrm{NMDA}}(t)\right), \\
\frac{\mathrm{d} x(t)}{\mathrm{d} t}=-\frac{x(t)}{\tau_{\mathrm{NMDA}, \text { rise }}}+\sum_{k} \delta\left(t-t^{k}\right), \\
\frac{\mathrm{d} s_{\mathrm{GABA}}(t)}{\mathrm{d} t}=-\frac{s_{\mathrm{GABA}}(t)}{\tau_{\mathrm{GABA}}}+\sum_{k} \delta\left(t-t^{k}\right),
\end{gathered}
$$

where $\delta\left(t-t^{k}\right)$ is the Dirac delta function, which represent the spikes emitted by $k$-th presynaptic neuron at the time $t^{k}$. In this work, the value of $\alpha$ is set as $\alpha=0.74 \mathrm{~m} \cdot \mathrm{s}^{-1}$. It is noted that the rise time for NMDA-mediated and GABAmediated synapses are not modeled in this work because the rise time $(<1 \mathrm{~ms})$ is very short, which is much smaller than the decay time [16]. The decay constants $\tau_{\mathrm{AMPA}}=2 \mathrm{~ms}, \tau_{\mathrm{NMDA} \text {,decay }}=100 \mathrm{~ms}$, and $\tau_{\mathrm{GABA}}=10 \mathrm{~ms}$ for $\mathrm{AMPA}$, NMDA and GABA synapses respectively. The rise time for NMDA synapses is set as $\tau_{\mathrm{NMDA}, \text { rise }}=2 \mathrm{~ms}$ [33].

The values of synaptic conductances for pyramidal neurons are set as follows: $g_{\mathrm{AMPA}, \mathrm{ext}}=2.08 \mathrm{nS}, g_{\mathrm{AMPA}, \mathrm{rec}}=0.104 \mathrm{~ns}, g_{\mathrm{NMDA}}=0.328 \mathrm{~ns}$, and $g_{\mathrm{GABA}}=$ $1.44 \mathrm{~ns}$. For inhibitory interneurons, the values of synaptic conductances are given as: $g_{\mathrm{AMPA}, \text { ext }}=1.62 \mathrm{~ns}, g_{\mathrm{AMPA}, \mathrm{rec}}=0.081 \mathrm{~ns}, g_{\mathrm{NMDA}}=0.258 \mathrm{~ns}$, and $g_{\mathrm{GABA}}=$ $0.973 \mathrm{~ns}$. $g_{\mathrm{AMPA}, \mathrm{ext}}$ is the synaptic efficacy from the outside input to all neurons; $g_{\mathrm{AMPA}, \mathrm{rec}}$ is the conductance mediate the excitatory recurrent current. In this work, the second-order Runge-Kutta method is used to solve the synaptic differential equation, and $\mathrm{d} t=0.1 \mathrm{msec}$ is applied for integration step.

\section{Results}

This paper uses MATLAB coding to implement the model. The computing devices used are configured as: memory (16G), CPU (Intel Xeon E5-1620). We first check the performance of the sequence memory in the model. In order to realized the sequential activation of the specific coding pools $P_{n}$, a global inhibitory signal for all specific coding pools is introduced in this work. Based on proposed model, a sequence recall in the sequence meomory modular is shown in Fig. 2(a). The example in Fig. 2(a) is formed by four items. Fig. 2(a) shows the rate of average activity in the four specific pools corresponding to the items in the sequence. After each switch, the next item is activated, and the previous item is inhibited. The two choice unconscious decision making without up-down control is presented in Fig. 2(b). In Fig. 2(b), there is no up-down control from the sequence recall. There are two optional decisions in Fig. 2(b), and the each decision component receives external stimulus just like saccade test. The excitation coding pool for each decision 


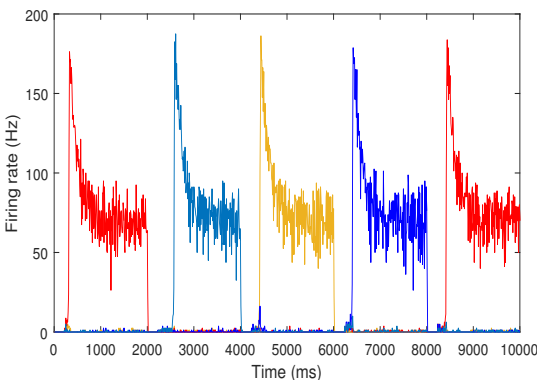

(a)

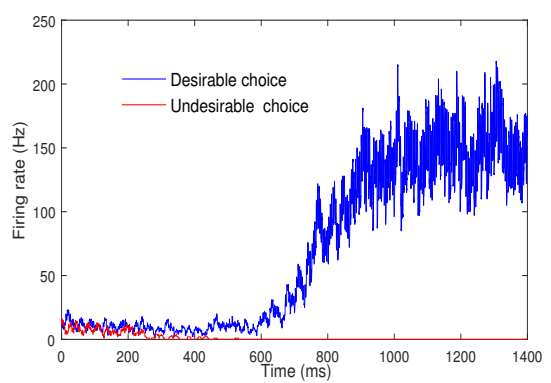

(b)

Fig. 2 (a) The sequence memory with four patterns, and each color indicates a pattern; (b) Two choice unconscious decision making without up-down control.

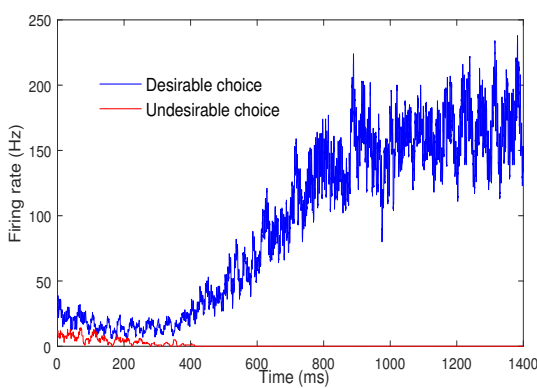

(a)

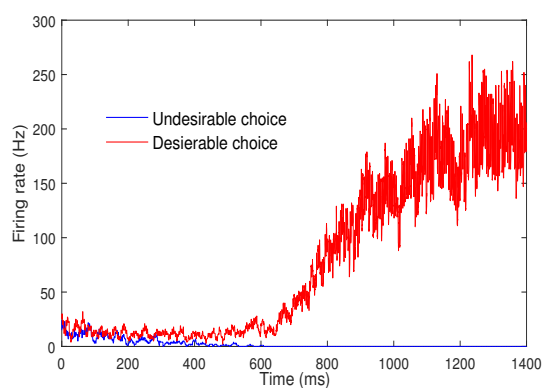

(b)

Fig. 3 (a) Two choice decision making sustained excitation by one memory pattern, which has same desirable decision with that of the unconscious; (b) Two choice decision making sustained excitation by one memory pattern, which has different desirable decision with that of the unconscious.

compete with each other, and one is activated, and another is suppressed. The simulation indicates that the decision time is more than $600 \mathrm{~ms}$.

Fig. 3 gives the process of network decision making under persistent up-down control signals. Fig. 3(a) and Fig. 3(b) receive two different external stimulus respectively. Fig. 3(a) shows two choice decision making which has same desirable decision with that of the automatic responses, while Fig. 3(b) shows two choice decision making which is different from that of the automatic responses. It can be seen from Fig. 3 that if the stimulus response and conscious decision-making are consistent, then the decision time will be relatively short, and the decision can be made in about $400 \mathrm{~ms}$. The simulation indicates that the decision time can be shortened if the stimulus response and the conscious decision are inconsistent. If the stimulus response and the conscious decision are inconsistent, then the decision time is about 600 milliseconds, which is same as the stimulus response time in Fig. 2(b). In fact, the weight of the external stimulus is less than the weight 
between the memory module and the decision pool. In Fig. 3, the intensity of the external stimulus is less than that of the memory stimulus. That's why the brain is able to make conscious decisions in Fig. 3(b). For the simulation of Fig. 3(b), if the intensity of external stimuli is large enough, automation responses will occur, and memory based decisions will be inhibited.

In order to reflect the dynamic process of network in multiple decision making, Fig. 4 gives the decision results under different conditions with three optional decisions. Fig. 4(a) shows the automation response with one choice in three options, and there is no up-down control signal on decision pools. Fig. 4(b) presents conscious decision making with one choice in three options sustained excitation by one memory pattern, which has same desirable decision with that of the automation response. The simulation results indicate that the decision time is shortened when the up-down control signal has same desirable decision with that of the automation response. Fig. 4(c) shows the automation response with two choice in three options, and there is no up-down control signal on decision pools. Fig. 4(d) presents the conscious decision making with two choice in three options sustained excitation by one memory pattern, which has same desirable decisions with that of the unconscious. Fig. 4(c)(d) are the multiple decision making. In reality, a memory pattern may correspond to multiple actions. Fig. 4(e) shows the conscious decision making with two choice in three options sustained excitation by one memory pattern, which has one desirable decision same as that of the unconscious, and another is different from the the unconscious. Although there is a pattern of conscious decision making and the pattern of stimulus response is inconsistent, two decisions have same decision time, which are about $400 \mathrm{~ms}$. Fig. 4(f) shows the conscious decision making with two choice in three options sustained excitation by one memory pattern, which has different decision from that of the unconscious. The simulation in Fig. 4(f) indicates that the decision time of two choices is same, it is about $600 \mathrm{~ms}$. From Fig. 4(e)(f), it can be seen that the decision time of all decision patterns is consistent in multi-mode decision making in any case. That is, when making conscious decision, no matter how many decisions are made, it is realized simultaneously.

Fig. 5 shows the sequence multiple conscious decision making based on the sequence memory. Fig. 5(a) gives the transformation between single decision patterns, which occurs when the patterns of sequential memory change. The decision patterns are corresponding to different memory patterns. It can be seen from Fig. 5(a) that when the conscious decision making and the autonomic response are consistent, the decision making speed is faster, about $400 \mathrm{~ms}$. At $1800 \mathrm{~ms}$, the first sequential memory pattern ends, and the memory pattern corresponding to the decision pattern will also be inhibited by the inhibition pool. At about $2400 \mathrm{~ms}$, the second memory pattern emerges, and the second memory pattern stimulates the second decision pattern. Fig. 5(b) gives the transformation between a single decision pattern and multiple decision patterns. The first pattern of sequential memory corresponds to the first decision pattern, and the second memory pattern of sequential memory correspond to two decision patterns. It can be seen from Fig. 5(b) that when a memory pattern corresponds to multiple decision patterns, the average firing rate of each decision pool will be reduced by half when compared to a single decision pattern. From the perspective of cognitive neuroscience, the 
Min Xia: Memory based decision making: A spiking neural circuit model

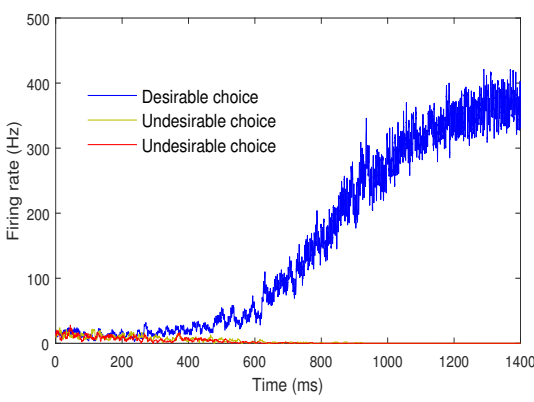

(a)

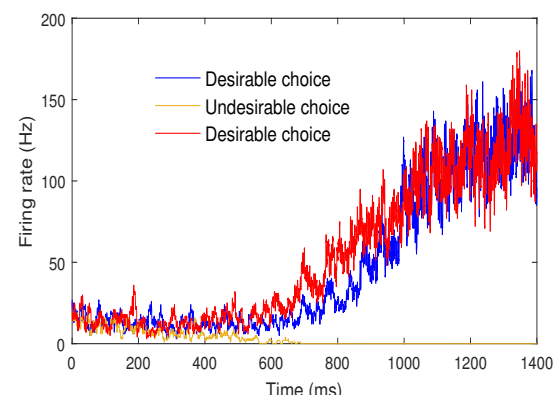

(c)

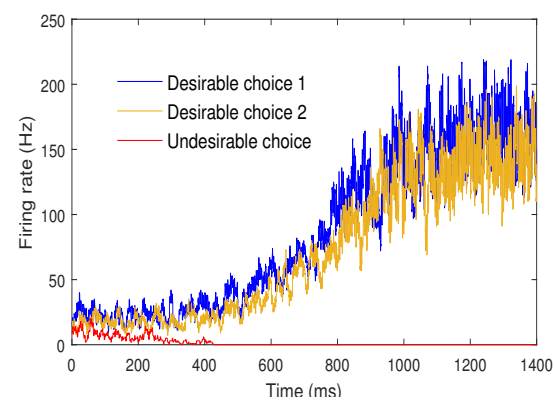

(e)

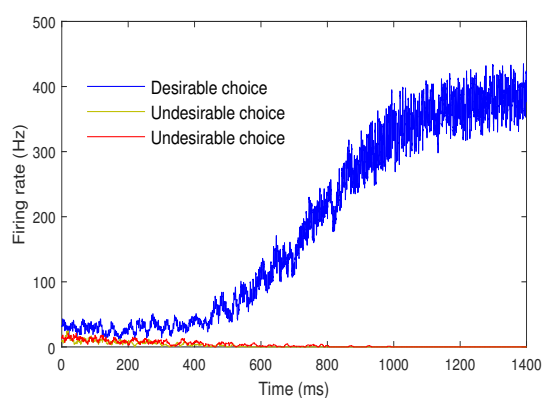

(b)

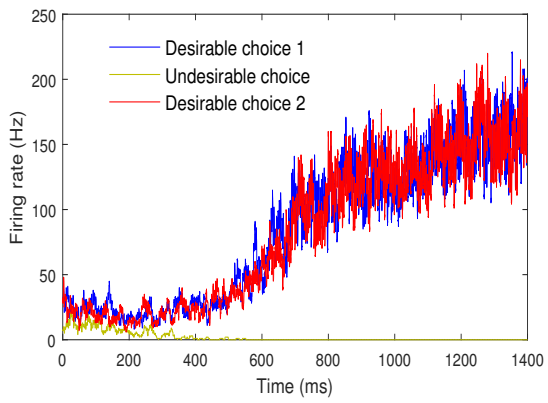

(d)

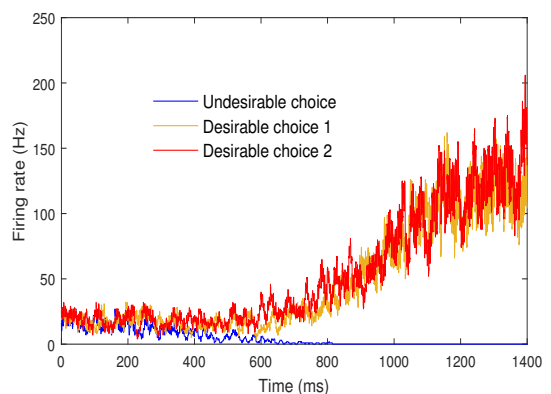

(f)

Fig. 4 (a)Unconscious decision making with one choice in three options; (b) Decision making with one choice in three options sustained excitation by one memory pattern, which has same desirable decision with that of the unconscious; (c) Unconscious decision making with two choice in three options; (d) Decision making with two choice in three options sustained excitation by one memory pattern, which has same desirable decisions with that of the unconscious; (e) Decision making with two choice in three options sustained excitation by one memory pattern, which has one desirable decision same as that of the unconscious, and another is different from the the unconscious; (f) Decision making with two choice in three options sustained excitation by one memory pattern, which has different decision from that of the unconscious. 


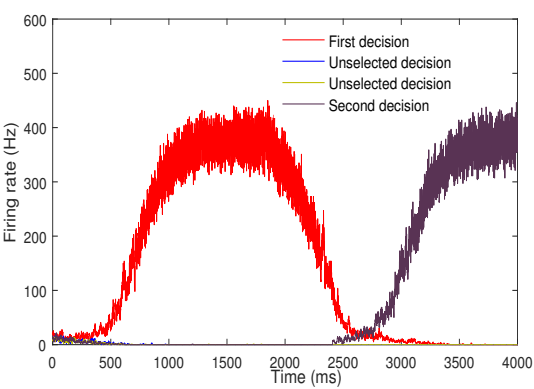

(a)

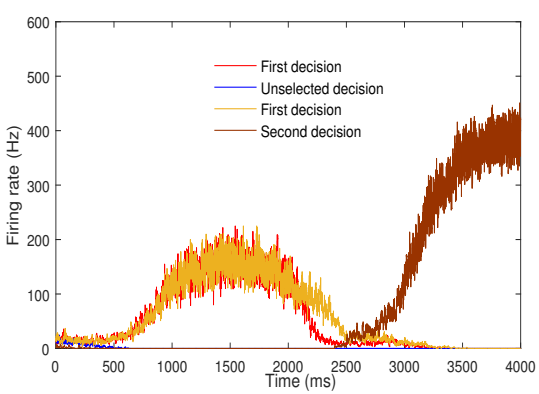

(c)

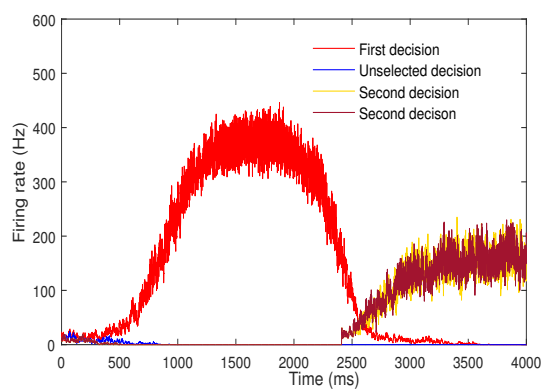

(b)

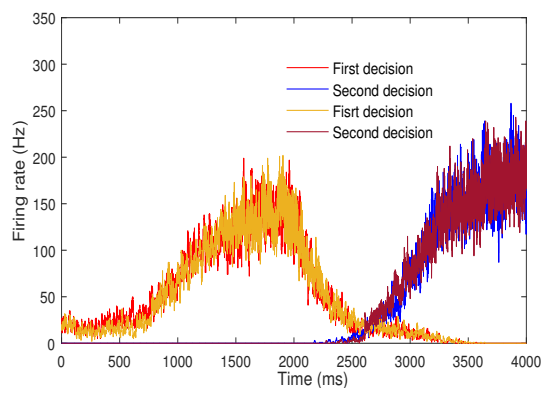

(d)

Fig. 5 Sequence multiple conscious decision making based on the sequence memory. (a)From single decision pattern to another decision pattern, all the conscious decisions are same as unconscious decision; (b)From single decision pattern to another two decision patterns, all the conscious decisions are same as unconscious decision; (c) From two decision patterns to another pattern, all the conscious decisions are different from unconscious decision; (d)From two decision patterns to another two patterns, the first decisions are different from unconscious decision, and the second decisions are same as unconscious decision.

brain's ability to respond is limited. Therefore, when a memory pattern corresponds to multiple simultaneous decision making, the reactivity of each decision will be reduced, and the model proposed in this paper can well prove this point. Fig. 5(c) gives a jump from two decision patterns to another decision pattern, and all the conscious decisions are different from unconscious decision. Assuming the external stimulus to unconscious pool in the experiment time is continuous. That is, external stimuli have been acting on unselected decision pool persistently. It can be seen from simulation that for the first decision, the decision time is relatively long, about $600 \mathrm{~ms}$, while the decision time is very short for the second decision models. When the first memory pattern disappears, such as $2000 \mathrm{~ms} 2500 \mathrm{~ms}$ in Fig. 1, the decision pattern corresponding to the memory will be inhibited by the inhibitory pool, and the firing rate will gradually decrease, but in the process, the first decision pattern can still inhibit the occurrence of the unconscious response. Therefore, unselected decision making pool is always resting, and can not stimulate 
other neurons. Thus, the decision time of the second decision pattern in Fig. 5(c) is not affected by the external stimulus signals. Fig. 5(d) gives the transition from multiple decision patterns to multiple decision patterns, and the first decisions are different from unconscious decision, the second decisions are same as unconscious decision. The simulation shows that the average firing rate of the second decision patterns is higher than the first decision patterns. This is because the second memory pattern and external stimuli have the same decision patterns, and the two signals act on second decision patterns, which results in increased stimulus intensity.

\section{Conclusion}

Brain decision making is one of the most important neural activities in human beings. Automatic response and conscious decision making are two important representations of brain decision-making. The flexibility of behavior activity is an important sign of human brain decision-making ability, which requires the brain to solve the conflict between automatic response and conscious decision-making. The current research focuses on the expression of decision information, but rarely involves the transmission mechanism of conscious decision-making information. This paper establishes a memory based conscious decision-making model, in which the memory pattern of sequential memory is used as the top-down control signal, and compete with the automatic response, so as to realize the conscious decision-making. In addition, the proposed model considers the multi-patterns decision making, and the reaction intensity decision pattern decreases with the increase of number of decision patterns. The reliability of the model is verified by simulation experiments.

From the physiological point of view, the memory signal should be mediated by the basal ganglia. The choice of decision making is realize by the inhibitory effect of neural circuits in basal ganglia [18]. This paper does not consider the conduction of the basal ganglia. Recent neural results have found that feedback from the external segment of globus pallidus to the striatum in the basal ganglia is critical for inhibitory control, and this feedback effect can realize the interdependence between memory and inhibitory control $[39,40]$. Moreover, the indirect pathway composed of striatum, globus pallidus, subthalamic nucleus STN and substantia nigra pars recitulata (SNr) can balance the inhibitory effect of direct pathway. These results are in urgent need of the biological neural dynamics mechanism model to explain the related phenomenon. In the future works, the modeling of the basal ganglia should be considered in the decision model.

\section{Acknowledgement}

This work is supported in part by the National Natural Science Foundation of PR China (No. 61773219), Natural Science Foundation of Jiangsu Province (BK20161533), and Qing Lan Project of Jiangsu Province. 


\section{Neural Network World 3/2019, 135-149}

\section{References}

[1] KUO W., SJOSTROM T., CHEN Y., et al. Intuition and deliberation: two systems for strategizing in the brain. Science. 2009, 324(5926), pp. 519-522, doi: 10.1126/science. 1165598.

[2] WEI H., BU Y., DAI D. A decision-making model based on a spiking neural circuit and synaptic plasticity. Cognitive Neurodynamics. 2017, 11(5), pp. 415-431, doi: 10.1007/ s11571-017-9436-2.

[3] GIRE D., WHITESELL J., DOUCETTE W., et al. Information for decision-making and stimulus identification is multiplexed in sensory cortex. Nature Neuroscience. 2013, 16(8), pp. 991-993, doi: 10.1038/nn.3432.

[4] WIMMER G., BCHEL C. Reactivation of Reward-Related Patterns from Single Past Episodes Supports Memory-Based Decision Making. J Neuroscience. 2016, 36(10), pp. 28682880.

[5] FENG N., YAO Y. No rounding reverse fuzzy morphological associative memories. Neural Network World. 2016, 26(6), pp. 571-587.

[6] XIA M., WENG L., WANG Z., et al. Sequence memory based on an oscillatory neural network. Science China-information Sciences. 2014, 57(7), pp. 1-12, doi: 10.7554/eLife. 04580.001.

[7] CHUNG L., WANG X. Conflict Resolution as Near-Threshold Decision-Making: A Spiking Neural Circuit Model with Two-Stage Competition for Antisaccadic Task. Plos Computational Biology. 2016, 12(8), pp. e1005081.

[8] CUTSURIDIS V., KUMARI V. Antisaccade performance in schizophrenia: a neural model of decision making in the superior colliculus. Frontiers in Neuroscience. 2014, 8, pp. 13, doi: 10.3389/fnins.2014.00013.

[9] SVITEK M. Towards complex system theory, Tutorial. Neural Network World. 2015, 25(1), pp. 5-33, doi: 10.14311/NNW.2015.25.001.

[10] WANG X. (2002) Probabilistic Decision Making by Slow Reverberation in Cortical Circuits. Neuron. 2002, 36(5), pp. 955-968, doi: 10.1016/S0896-6273(02)01092-9.

[11] MACHENS C., ROMO R., BRODY C. Flexible control of mutual inhibition: a neural model of two-interval discrimination. Science. 2005, 307(5712), pp. 1121-1124, doi: 10.1126/ science.1104171.

[12] DECO G., ROLLS E. Decision-making and Weber's law: a neurophysiological model. European Journal of Neuroscience. 2006, 24(3), pp. 901-916, doi: 10.1111/j.1460-9568.2006. 04940.x.

[13] KOSTER H. A microcircuit model of the frontal eye fields. Journal of Neuroscience. 2007, 27(35), pp. 9341-9353.

[14] CAIN N., SHEABROWN E. Impact of correlated neural activity on decision-making performance. Neurocomputing. 2013, 25(2), pp. 289-327, doi: 10.1.1.593.8655.

[15] THURA D., COS I., TRUNG J., et al. Context-dependent urgency influences speed-accuracy trade-offs in decision-making and movement execution. journal of neuroscience. 2014, 34(49), pp. 16442-16454, doi: 10.1523/JNEUROSCI.0162-14.2014.

[16] LO C., WANG C., WANG X. Speed-accuracy tradeoff by a control signal with balanced excitation and inhibition. Journal of Neurophysiology. 2015, 114(1), pp. 650-661.

[17] SPERA E., MIGLIORE M., UNSWORTH N., et al. On the cellular mechanisms underlying working memory capacity in humans. Neural Network World. 2016, 26(4), pp. 335-350, doi: 10.14311/NNW.2016.26.019.

[18] HIKOSAKA O., ISODA M. Switching from automatic to controlled behavior: cortico-basal ganglia mechanisms. Trends in Cognitive Sciences. 2010, 14(4), pp. 154-161, doi: 10.1016/ j.tics.2010.01.006.

[19] FECHNER H., PACHUR T,. SCHOOLER L., MEHLHORN K., BATTAL C., VOLZ K., et al. Strategies for memory-based decision making: Modeling behavioral and neural signatures within a cognitive architecture. Cognition. 2016, 157, pp. 77-99. 
[20] KHADER P., PACHUR T., WEBER L., et al. Neural Signatures of Controlled and Automatic Retrieval Processes in Memory-based Decision-making. Journal of Cognitive Neuroscience. 2016, 28(1), pp. 69-83.

[21] BOGACZ R., LARSEN T. Integration of reinforcement learning and optimal decision-making theories of the basal ganglia. Neurocomputing. 2011, 23(4), pp. 817-851.

[22] WEI W., RUBIN J., WANG X. Role of the Indirect Pathway of the Basal Ganglia in Perceptual Decision Making. Journal of Neuroscience. 2015, 35(9), pp. 4052-4064, doi: 10.1523/JNEUROSCI.3611-14.2015.

[23] XIA M., WONG W., WANG Z. Sequence memory based on coherent spin-interaction neural networks. Neurocomputing. 2014, 26(12), pp. 2944-2961.

[24] BRUNEL N., WANG X. Effects of neuromodulation in a cortical networks model of object working memory dominated by recurrent inhibition. Journal of Computational Neuroscience. 2001, 11, pp. 63-85, doi: 10.1007/s10827-014-0506-8.

[25] ALEXANDER R., GIANCARLO L.C., LUSCHER H., et al. Neocortical Pyramidal Cells Respond as Integrate-and-Fire Neurons to In Vivo-Like Input Currents. Journal of Neurophysiology. 2003, 90(3), pp. 1598-1612, doi: 10.1152/jn.00067.2004.

[26] DECO G., ROLLS E. Sequential Memory: A Putative Neural and Synaptic Dynamical Mechanism. Journal of Cognitive Neuroscience. 2005, 17(2), pp. 294-307.

[27] ROLLS E., DECO G. Stochastic cortical neurodynamics underlying the memory and cognitive changes in aging. Neurobiology of Learning and Memory. 2015, 118, pp. 150, doi: $10.1016 / \mathrm{j} . \mathrm{nlm} .2014 .12 .003$.

[28] WANG Y., ROMANI S., LUSTIG B., et al. Theta sequences are essential for internally generated hippocampal firing fields. Nature Neuroscience. 2014, 18(2), pp. 282-288, doi: 10. 1073/pnas. 1703309114

[29] COMPte A., CONSTANTINIDIS C., TEgNER J., RAGHAVACHARI S., CHAFEE M. GOLDMAN-RAKIC P., WANG X. Temporally irregular mnemonic persistent activity in prefrontal neurons of monkeys during a delayed response task. Journal of Neurophysiology. 2003, 90, pp. 3441-3454, doi: 10.1152/jn.00949. 2002.

[30] ABELES A. Corticonics. 1991, New York, Cambridge University Press.

[31] MOOS P., SVITEK M., NOVAK M., VOTRUBA Z. Information Model of Resonance Phenomena in Brain Neural Networks. Neural Network World 2018, 28(3), pp. 225-239, doi: $10.14311 / \mathrm{NNW} .2018 \cdot 28.014$

[32] SHADlEn M., SHOHAMY D. Decision Making and Sequential Sampling from Memory. Neuron, 2016, 90(5), pp. 927-939, doi: 10.1016/j.neuron.2016.04.036.

[33] PEREIRA J., WANG X. A Tradeoff Between Accuracy and Flexibility in a Working Memory Circuit Endowed with Slow Feedback Mechanisms. Cerebral Cortex. 2015, 25(10), pp. 3586.

[34] ROITMAN J., SHADLEN M. Response of neurons in the lateral intraparietal area during a combined visual discrimination reaction time task. Journal of Neuroscience. 2002, 22, pp. 9475-9489.

[35] MACHENS C.K. NEUROSCIENCE. Building the human brain. Science, 2012, 338(6111), 1156, doi: 10.1126 /science.1231865.

[36] GALLIVAN J.P., BOWMAN N.A.R., CHAPMAN C.S., et al. The sequential encoding of competing action goals involves dynamic restructuring of motor plans in working memory. Journal of Neurophysiology. 2016, 115(6), pp. 3113-3122.

[37] LO C., WANG X.Cortico-basal ganglia circuit mechanism for a decision threshold in reaction time tasks. Nature Neuroscience. 2006, 9(7), pp. 956-963.

[38] JAHR C., STEVENS C. Voltage dependence of NMDA-activated macroscopic condunctances predicted by single-channel kinetics. Journal of Neuroscience. 1990, 10, pp. 3178-3182.

[39] BENUSKOVA L., JEDLICKA P. Computational modeling of long-term depression of synaptic weights: insights from STDP, metaplasticity and spontaneous activity. Neural Network World. 2012, 22(2), 161-180, doi: 10.14311/NNW.2012.22.010.

[40] WEI W., WANG X. Inhibitory Control in the Cortico-Basal Ganglia-Thalamocortical Loop: Complex Regulation and Interplay with Memory and Decision Processes. Neuron. 2016, 92(5), pp. 1093, doi: 10.1016/j.neuron.2016.10.031. 